\title{
Field-based sciences must transform in response to COVID-19
}

The pandemic will allow us to fundamentally remodel the way field-based sciences are taught, conducted and funded - but only if we stop waiting for a 'return to normal'.

\author{
Eleanor M. L. Scerri, Denise Kühnert, James Blinkhorn, Huw S. Groucutt, Patrick Roberts, Kathleen Nicoll, \\ Andrea Zerboni, Emuobosa Akpo Orijemie, Huw Barton, lan Candy, Steven T. Goldstein, John Hawks, \\ Khady Niang, Didier N'Dah, Michael D. Petraglia and Nicholas C. Vella
}

W e study human origins from the related disciplines of archaeology, palaeoanthropology and allied geosciences, which are driven forward by making new discoveries in the field. Collectively, our scientific ecosystem informs climate change policy, heritage management and conservation practices. The pandemic has forced an inevitable pause on our work, but several scientific studies now indicate that the severe acute respiratory syndrome coronavirus 2 (SARS-CoV-2), the causative agent of coronavirus disease 2019 (COVID-19), may be here to stay ${ }^{1-6}$. Critically for fieldwork and related travel considerations, the pandemic will increasingly display temporally and spatially fragmented peaks and troughs? Complicating this is the fact that affected countries are not consistent in their public health decisions and application of World Health Organization (WHO) guidance ${ }^{8}$, particularly in terms of easing containment measures. Regional and country-based variations in the availability and quality of appropriate healthcare also underline the importance of protecting at risk communities.

Given these projections, a good, ethical model for 'remote science' is essential. So far, however, we have seen only short-term mitigation strategies, such as online meetings, postponement of fieldwork and short grant extensions ${ }^{9}$. These solutions are fundamentally oriented towards patching up a working model whose practices have grown around cheap, fast and relatively unrestricted travel and face-to-face interactions - and thus assume an eventual return to pre-pandemic normality. However, the effects of the crisis cannot simply be waited out. Instead, we need to radically reshape the way we operate in science. Chief among the required changes is the urgent need for alternative ways to interact that require less travel - a goal long-advocated for by the movement to decarbonize field

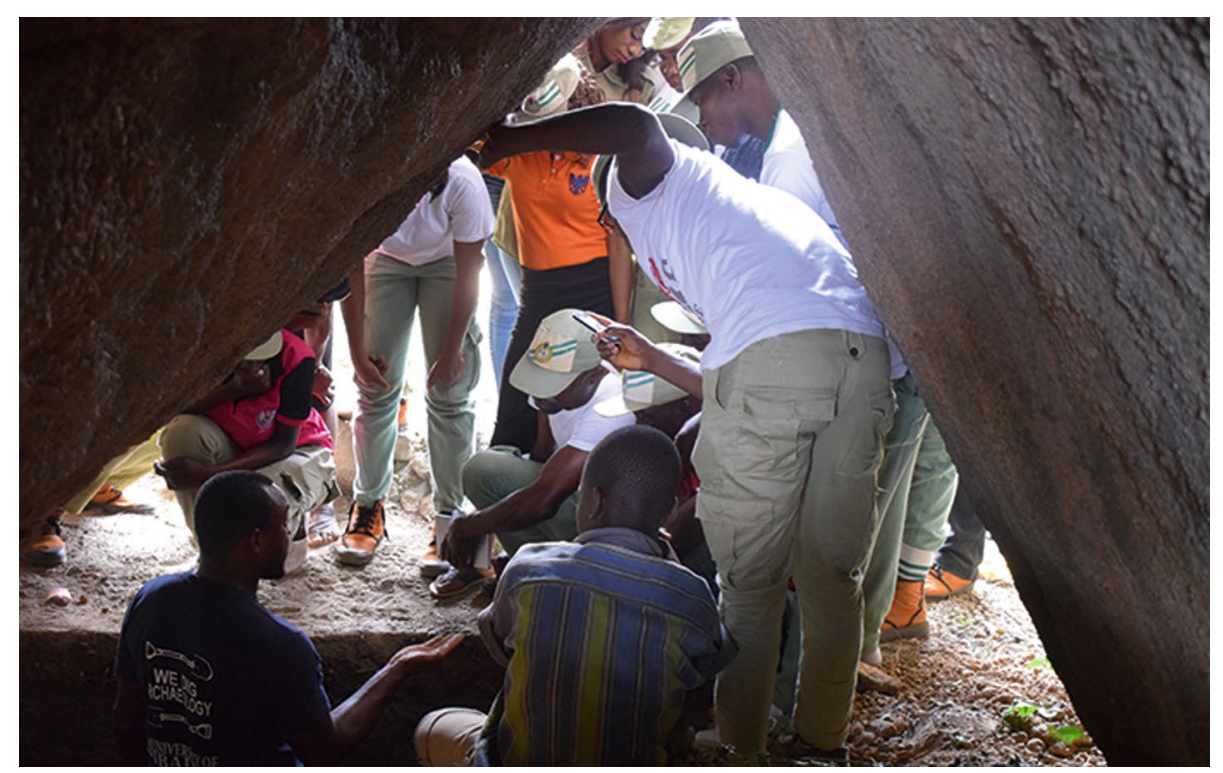

Fig. 1 | Community archaeology in Nigeria led by co-author E. A. Orijemie, far left. Following practices described in the main text, this programme is resuming while negotiating remote collaborative procedures with international collaborators.

sciences - as well as ensuring equitable research partnerships and the elimination of nominal collaborations that overwhelmingly favour the career advancement of partners in the Global North. Here we articulate solutions to achieve these goals.

\section{Step 1: Ethical considerations}

Ethical considerations lie at the heart of the many fieldwork-related issues raised by the pandemic. With increasing geographic disparities in COVID-19 epidemiology, how do we decide whether, when and how field research can be initiated or resumed? Institutions not only need to consider their own ethical obligations, but ensure that their agents and partners also take such obligations seriously. For example, what should be done if rules or norms in a given locality provide too little protection for researchers and other stakeholders, including local communities? Is protection adequate for other pathogens, given that COVID-19 has put the brakes on programmes to contain other diseases ${ }^{10}$ ? How do institutions prevent situations in which individuals attempt to pursue incentives to resume fieldwork sooner than is ethically advisable? Besides addressing these questions, international partners must ensure leadership is given to in-country research collaborators at field site locations. This is particularly the case for international collaborations across the Global North-South divide. A duty of care should be distinguished from colonialist legacies in which the 'burden' of decision making is appropriated by institutions in the Global North in the name of safety ${ }^{11}$. 


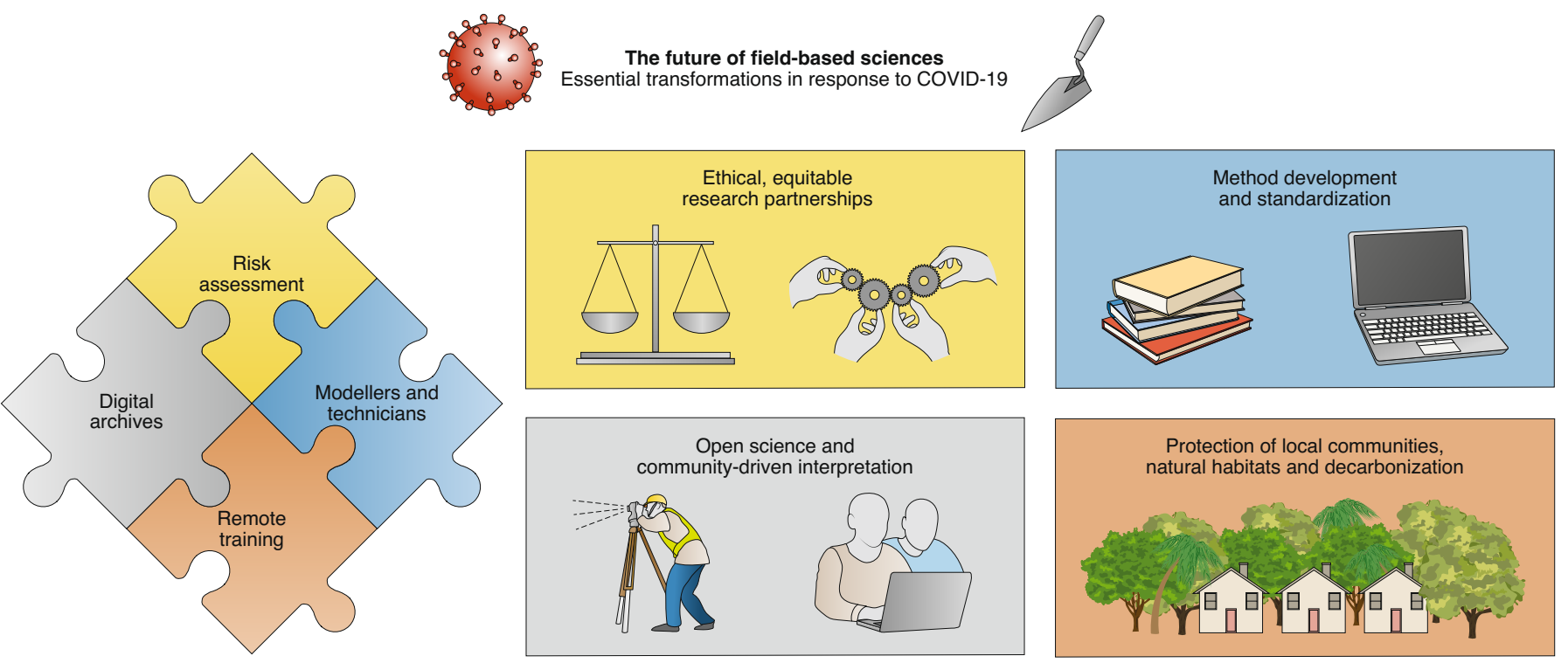

Fig. 2 | Essential pieces of the puzzle to transform field-based sciences in response to COVID-19. Remote science requires risk assessments to facilitate equitable research partnerships (yellow), investment in technicians and modellers to advance method development, standardization and to cope with post-processing demands (blue), remote training to protect local communities and decarbonize fieldwork (orange), and ultimately, the creation of digital archives to move towards open-science and community inference (grey).

Comprehensive evaluation must involve a stepped risk assessment. Independent virtual consultation groups, featuring scholars whose local, regional and global disciplinary expertise should be involved to inform decision making as part of risk assessment processes. Such consultation panels should provide recommendations that can then precipitate assessment adjusted to the specificities of the situation and project. Core considerations should include: (1) the need to balance the safety of all stakeholders, research possibilities and future risks to our disciplines; (2) ensuring diverse perspectives are taken into consideration; and (3) where relevant, generally situating decision making at the location of data, not the location of the principal investigator.

To facilitate community shielding, a transparent and documented risk assessment procedure must also be coupled with new technologies such as contact tracing apps ${ }^{12}$, greater delegation and decision-sharing practices within international and interregional partnerships. Ensuring chains of communication remain safely active will be challenging, but these are indispensable. Investment in local communities is key for successful remote collaboration. Such investment delegates and distributes monitoring and heritage management among local community stakeholders, following practices already common in ethnography (for example, refs. ${ }^{11,13}$ ) (Fig. 1).

\section{Step 2: Technological solutions}

When only local participation in fieldwork is possible, how effectively can remote collaborations be executed in the field sciences, when so much diverse expertise is required?

Foremost is a comprehensive investment in the creation of digital archives at different scales. Various government agencies and developer-led fieldwork as well as excavations in extreme locations have been using such methods and techniques for years ${ }^{14}$. However, practice is neither standardized nor mainstreamed across comparable research projects, and in the context of COVID-19 there are many reasons to push for such goals.

First, the creation of high-resolution photographic databases for photogrammetry is relatively easy to teach remotely, and is inexpensive, although post-processing time is substantial and requires investment in technicians. These techniques can record and visualize spatial relationships, stratigraphic sequences and, depending on the use of different light, may permit assessments that can even be superior to traditional by-eye illustrations ${ }^{14}$. Specialists can clearly mark sampling locations on the models for those on site, enabling group assessments. Second, the creation of three-dimensional (3D) geological and sedimentary archives also enables re-assessment of sequences in future. Third, the creation and sharing of both the archives and their interpretations will precipitate the much-needed standardization of sampling and analytical procedures. Within an open data framework, this working model will ensure that novice researchers and non-specialists learn from experts through collaborative, team-based inferences, rather than stitching together the results of individual specialists in a top-down approach.

Our existing international field season schedules also require change - a long-overdue adjustment in the face of increasing anthropogenically induced climate change. Fieldwork will be based locally and projects will require many short and closely spaced field seasons. Those who can access our field sites within a few hours can conduct short-distance trips, focused on discrete steps in the process of assessment, excavation, sampling and inference. For example, a short initial season would focus on building a high-resolution digital model of the field site that can be shared with remote collaborators to develop excavation strategies. A later season could focus solely on sampling, following remote collaborative assessment of digital archives. Such approaches also in part mitigate the problems faced by less-accessible field sites, where frequent online meetings and the exchange of information are impossible. Effective remote collaboration will require very clear scheduling among remote experts at each phase of the process to minimize the burden on local researchers. 
Box 1 | Funding agencies need to prioritize funding in four areas

1. Experimental and method development work, for example exploring the inferential power of $3 \mathrm{D}$ archives versus traditional by-eye onsite inferences from specialists. 2. Large, gatekeeper-free database initiatives. At the very least, this ensures the continuation of the spirit and act of collaboration and interaction that is so essential to the fieldwork experience.

3. The $3 \mathrm{D}$ digitization and curation of museum archives and collections. This will not only protect valuable collections from future challenges, but also mitigate the need to travel. Funders should recognize

At a landscape scale, the situation becomes more challenging. 3D models from unmanned aerial vehicles (UAVs), remote-sensing data ${ }^{15}$ and $\mathrm{LiDAR}^{16}$ are already widely used in prospection and analysis, and may facilitate effective collaboration between remote specialists and local participants. Remotely generated landscape-scale hypotheses and geomorphological maps can be tested at a later stage by specialists on the ground, whenever longer-distance travel becomes an appropriate option. The creation of $3 \mathrm{D}$ data using UAVs does not represent a considerable remote training challenge. A major new research focus should address the extent to which these methods and predictive models in geomorphology are able to replicate, complement and validate assessments of landscape-scale processes made in the field.

\section{Training and students}

Technological solutions to research problems do not exist within a vacuum. Shifts in methodologies require important shifts in the way we teach. The loss of fieldwork can critically endanger the completion of a graduate research project and, for undergraduate students, there is a looming risk that foundational field techniques cannot be taught during this pandemic. This threatens core elements of student training that are vital to academic and career development. Onsite training in field methods, such as archaeological survey and excavation, are fundamental to our disciplines, leading to the hard-learned intellectual practice of revealing and conceptualizing the spatio-temporal relationships of materials, events and landscapes. Universities and research institutions implementing alternatives to field-based projects are to be applauded, but the loss of bench fees and other means of support for institutions in the Global South, and develop new sustainable partnerships.

4. Technicians and assistants for enabling access, processing and storing large amounts of digital information. Currently, funding programmes prioritize research staff over technical staff where it may not always be appropriate to do so. The important role of technical staff and assistants must be recognized and financially supported in a viable manner; they should not be viewed as an easy way to cut costs.

these are short-term solutions and provide little mitigation for future students in the long term.

One obvious solution has been to rely on existing data that have not yet been analysed, and the reformulation and creation of new datasets. Some platforms, such as Otlet, are attempting to engage with scientific matchmaking, in which biological scientists can connect with prospective students. Large databases such as IsoARCH and Neotoma enable new projects, for example through meta-analyses. Rolling out similar solutions more broadly across field-based sciences would undoubtedly make a difference.

However, these solutions do not compensate for the loss of field interpretation, sampling or analytical experience and will only provide solutions for a small number of years. It is therefore essential that students are exposed to digital archives and their interpretation. For example, teaching modules can creatively focus on comparing 3D archives with the actual sediments of small training excavations which are both local and permit physical distancing. Selecting appropriate sampling locations and correctly interpreting geomorphological processes should become part of the training arsenal of the next generation of field-based scientists (Fig. 2).

\section{Step 3: Institutional and funding agency changes}

The remodelling of fieldwork teaching and practice shines the spotlight on the need for change within institutional and funding agencies. Their existing reward structures have not yet confronted the potential ethical issues raised by a global pandemic, or the long-term challenges caused by ongoing climate change. The need to be flexible, provide funded extensions, reduce red-tape and transfer meetings to virtual platforms are all welcome measures. However, they only speak to the adjustments required for the completion of existing projects. For sustainable future projects, funding agencies need to stop prioritizing transformative discovery-focused science over method development, as the former carries greater scientific 'impact' in terms of resulting publications. A critical culture change is needed in the way funding agencies and scientific institutions work (see Box 1). Support is needed in the development of virtual methods for training students and early-career researchers, and project funding for testing the validity of virtual over traditional field methods is required. As many institutions also increasingly engage with virtual learning, such a lack of investment is increasingly out of sync with the state of the world and its future science and pedagogy.

Financially restructuring the critically important areas flagged in Box 1 will also drive equity and the fair geographic distribution of resources, because investing in the location of data, not the location of principal investigators, is more sustainable. This will ensure that infrastructure benefits are local to the areas, regions or countries where research is taking place. Furthermore, longstanding arguments by those in favour of Open Science must be more broadly accepted in a COVID-19 world ${ }^{17}$. Ensuring the future of field-based science requires greater investment in communication, shared analyses, open data repositories and international perspectives. In particular, accessible new digital archives will form an important part of peer-review and assessment, thus improving the documentary quality and reproducibility of scientific results.

This virus will probably affect our lives for many years, and novel viruses will continue to emerge as we continue to destroy animal habitats ${ }^{18,19}$. This means that field project closures and postponements will be unavoidable. By acting now, we can mitigate the severity of current and future impacts. We must be prepared to step outside our comfort zone and embrace changes that are, in some cases, long overdue. The COVID-19 crisis should prompt scientific communities to consider how technology can support better collaborations for both social and climate justice, as well as enable us to advance our work together, from a safe distance.

Eleanor M. L. Scerri (D) ${ }^{1 \times}$, Denise Kühnert (DD $2 \bowtie$, James Blinkhorn ${ }^{1,3}$, Huw S. Groucutt ${ }^{4,5,6}$, Patrick Roberts (D) , Kathleen Nicoll ${ }^{7}$, Andrea Zerboni ${ }^{8}$, 
Emuobosa Akpo Orijemie ${ }^{9}$, Huw Barton ${ }^{10}$, Ian Candy ${ }^{3}$, Steven T. Goldstein ${ }^{5}$, John Hawks ${ }^{11}$, Khady Niang ${ }^{12}$, Didier N'Dah ${ }^{13}$, Michael D. Petraglia ${ }^{5,14,15}$ and Nicholas C. Vella (D) 16

${ }^{1}$ Pan-African Evolution Research Group, Max Planck Institute for the Science of Human History, Jena, Germany. ${ }^{2}$ Transmission, Infection, Diversification \& Evolution Research Group, Max Planck Institute for the Science of Human History, Jena, Germany. ${ }^{3}$ Centre for Quaternary Research, Department of Geography, Royal Holloway, University of London, Egham, UK. ${ }^{4}$ Extreme Events Research Group, Max Planck Institute for Chemical Ecology, Jena, Germany. ${ }^{5}$ Department of Archaeology, Max Planck Institute for the Science of Human History, Jena, Germany. ${ }^{6}$ Max Planck Institute for Biogeochemistry, Jena, Germany. ${ }^{7}$ Department of Geography, University of Utah, Salt Lake City, UT, USA. ${ }^{8}$ Dipartimento di Scienze della Terra "A.Desio", Università degli Studi di Milano, Milano, Italy. ${ }^{9}$ Department of Archaeology and Anthropology, The University of Ibadan, Ibadan, Nigeria. ${ }^{10}$ School of Archaeology and Ancient History, University of Leicester, Leicester, UK. ${ }^{11}$ Department of Anthropology, University of Wisconsin-Madison, Madison, WI, USA. ${ }^{12}$ Département d'Histoire, Université Cheikh Anta Diop de Dakar, Dakar-Fann, Senegal. ${ }^{13}$ Department of History and Archaeology, University of Abomey-Calavi, Abomey-Calavi, Benin Republic. ${ }^{14}$ School of Social Science, University of Queensland, St Lucia, Queensland, Australia. ${ }^{15}$ Department of Anthropology, National Museum of Natural History, Smithsonian Institution, Washington DC, USA. ${ }^{16}$ Department of Classics and Archaeology, Faculty of Arts, University of Malta, Msida, Malta.

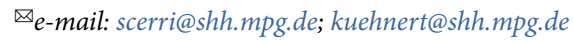

Published online: 14 September 2020 https://doi.org/10.1038/s41559-020-01317-8

References

1. Anderson, R. M., Heesterbeek, H., Klinkenberg, D. \& Hollingsworth, T. D. Lancet 395, 931-934 (2020).

2. Flodgren, G. M. Immunity after SARS-CoV-2 Infection, 1st Update A Rapid Review 2020 (Norwegian Institute of Public Health, 2020).

3. "Immunity Passports" in the Context of COVID-19. WHO

Scientific Brief (24 April 2020); https://go.nature.com/3cuLlqz

4. Gouglas, D. et al. Lancet Glob. Health 6, E1386-E1396 (2018).
5. Villar, J. et al. Lancet Respir. Med. 8, 267-276 (2020).

6. Low-cost dexamethasone reduces death by up to one third in hospitalised patients with severe respiratory complications of COVID-19. Oxford University News Release (16 June 2020); https://go.nature.com/2GiQ7MC

7. Kissler, S. M., Tedijanto, C., Goldstein, E., Grad, Y. H. \& Lipsitch, M. Science 368, 860-868 (2020).

8. WHO Technical Guidance (WHO, 2020); https://go.nature. $\mathrm{com} / 2 \mathrm{Z} 23 \mathrm{FCO}$

9. Stoye, E. How research funders are tackling coronavirus disruption. Nature News (17 April 2020); https://doi.org/10.1038/ d41586-020-01120-2

10. Roberts, L. 'We have no choice.' Pandemic forces polio eradication group to halt campaigns. Science News (1 April 2020); https://doi. org $/ 10.1126 /$ science.abc0263

11. Ogundiran, A. Afr. Archaeol. Rev. 37, 179-183 (2020).

12. Ferretti, L. et al. Science 368, eabb6936 (2020).

13. Broesch, T. et al. Preprint at PsyArXiv https://doi.org/10.31234/ osf.io/thqsw (2020).

14. Kruger, A., Randolph-Quinney, P. \& Elliott, M. S. Afr. J. Sci. 112, 2016-0032 (2016)

15. Breeze, P. S. et al. Quatern. Int. 382, 98-119 (2015).

16. Evans, D. J. Archaeol. Sci. 74, 164-175 (2016).

17. Marwick, B. et al. SAA Archaeol. Record 17, 8-14 (2017).

18. Jabr, F. How humanity unleashed a flood of new diseases. The New York Times (17 June 2020); https://go.nature.com/31S8sIW 19. Grubaugh, N. D. et al. Nat. Microbiol. 4, 10-19 (2019).

Competing interests

The authors declare no competing interests. 